\title{
A novel method for producing target cells and assessing cytotoxic $T$ lymphocyte activity in outbred hosts
}

\author{
Francesca Bonci ${ }^{\dagger}$, Elisa Zabogli ${ }^{\dagger}$, Francesca Conti, Antonio Merico, \\ Giulia Freer, Mauro Bendinelli and Mauro Pistello*
}

\author{
Address: Retrovirus Center and Virology Section, Department of Experimental Pathology, University of Pisa, Via San Zeno, 35, 56127 Pisa, Italy \\ Email: Francesca Bonci - f.bonci@kedrion.com; Elisa Zabogli - elisazabogli@biomed.unipi.it; Francesca Conti - francescaconti@biomed.unipi.it; \\ Antonio Merico - merico-antonio@libero.it; Giulia Freer - freer@biomed.unipi.it; Mauro Bendinelli - bendinelli@biomed.unipi.it; \\ Mauro Pistello* - pistello@biomed.unipi.it \\ * Corresponding author †Equal contributors
}

Published: II March 2009

BMC Biotechnology 2009, 9:18 doi:10.1 186/1472-6750-9-18
Received: 7 August 2008

Accepted: II March 2009

This article is available from: http://www.biomedcentral.com/1472-6750/9//8

(C) 2009 Bonci et al; licensee BioMed Central Ltd.

This is an Open Access article distributed under the terms of the Creative Commons Attribution License (http://creativecommons.org/licenses/by/2.0), which permits unrestricted use, distribution, and reproduction in any medium, provided the original work is properly cited.

\begin{abstract}
Background: Cytotoxic $T$ lymphocytes play a crucial role in the immunological control of microbial infections and in the design of vaccines and immunotherapies. Measurement of cytotoxic T lymphocyte activity requires that the test antigen is presented by target cells having the same or compatible class I major hystocompatibility complex antigens as the effector cells. Conventional assays use target cells labeled with ${ }^{51}$ chromium and infer cytotoxic $\mathrm{T}$ lymphocyte activity by measuring the isotope released by the target cells lysed following incubation with antigen-specific cytotoxic T lymphocytes. This assay is sensitive but needs manipulation and disposal of hazardous radioactive reagents and provides a bulk estimate of the reporter released, which may be influenced by spontaneous release of the label and other poorly controllable variables. Here we describe a novel method for producing target in outbred hosts and assessing cytotoxic T lymphocyte activity by flow cytometry.

Results: The method consists of culturing skin fibroblasts, immortalizing them with a replication defective clone of simian virus 40 , and finally transducing them with a bicistronic vector encoding the target antigen and the reporter green fluorescent protein. When used in a flow cytometrybased assay, the target cells obtained with this method proved valuable for assessing the viral envelope protein specific cytotoxic T lymphocyte activity in domestic cats acutely or chronically infected with feline immunodeficiency virus, a lentivirus similar to human immunodeficiency virus and used as animal model for AIDS studies.

Conclusion: Given the versatility of the bicistronic vector used, its ability to deliver multiple and large transgenes in target cells, and its extremely wide cell specificity when pseudotyped with the vesicular stomatitis virus envelope protein, the method is potentially exploitable in many animal species.
\end{abstract}




\section{Background}

Cytotoxic T lymphocytes (CTLs) are key components of the cell-mediated immune responses and play an essential role in protection from and containment of a variety of pathogens [1]. Since CTLs have been shown to play a major role in the control of human immunodeficiency virus (HIV) and other lentiviruses [2,3], measurement of their activity has become an important parameter for testing the efficacy of candidate vaccines and other immunologic interventions directed against these important agents of disease [4].

CTL assays require that the test antigen is presented by target cells having the same or compatible class I major hystocompatibility complex antigens as the effector cells. This permits to the antigen-specific CTLs to interact with the target cells and determine their lysis, which is then quantitated as a measure of CTL activity. The most used format is the ${ }^{51}$ chromium $\left({ }^{51} \mathrm{Cr}\right)$-release assay in which this radioisotope, absorbed by the target cells prior to mixing with the effector cells, is released in the supernatant medium and measured at the end of the incubation period [5]. This assay is sensitive but has the disadvantage of requiring the manipulation and disposal of hazardous radioactive reagents. Thus, over the years, ${ }^{51} \mathrm{Cr}$ has been tentatively replaced by various cell dyes with somewhat inconsistent results. However, all these assays have the limitation of providing a bulk estimate of the reporter released, which may be influenced by several, poorly controllable variables, such as inefficient labeling of target cells or spontaneous release of the label [6]. More recently, new flow cytometry-based (F-CTL) assays, using target cells labeled with fluorescent dyes or substrates for caspases or other apoptotic enzymes, have been proposed [7-12]. These assays appear to be as or more sensitive than the standard ${ }^{51} \mathrm{Cr}$ methods and, in addition, may permit to precisely count the target cells that have actually been lysed and to identify the phenotype of the effector cells involved $[6,9]$.

Here, we describe a novel method for producing target cells that has provided satisfactory results in an F-CTL assay we have set up to assess envelope (Env)-specific CTL activity in domestic specific pathogen-free (SPF) cats infected with feline immunodeficiency virus (FIV), the feline equivalent of HIV. Since no inbred cats are available, testing CTL activity in this species is dependent on the use of autologous target cells, a factor that has hitherto limited routine measurements of CTL activity $[13,14]$. The method relies on a bicistronic vector derived from FIV but having very little left of the original virus which is used to transduce immortalized feline skin fibroblasts. Since the vector can be pseudotyped with the different Envs and may have broad tropism [15], the method is potentially exploitable in other animal species as well.

\section{Results \\ Production and characterization of the target cells}

Production of the target cells expressing the Env of FIVPetaluma (FIV-Pet) and reporter green fluorescent protein (GFP) is schematically depicted by Figure 1. Primary fibroblasts were obtained by culturing skin biopsies of the study cats and, when confluent, transferred to larger flasks. One flask was then used to control for cell competence for transfection by using a plasmid encoding GFP (pcDNA3-GFP). As expected for primary cells and in agreement with Köksoy et al. [16], the fibroblasts proved moderately transfectable, since by day 2 post transfection GFP expressing cells ranged between 15\% and 30\% (data not shown). The remaining flasks were transfected with a plasmid encoding a replication defective simian virus 40 (SV40) genome (pACTSV2) and subcultured every 3-4 days for further 3 weeks. Ten to 15 days later, the pACTSV2 transfected cells showed unequivocal signs of immortalization that were maintained for at least 30 passages regardless of whether the cells were kept in culture continuously or repeatedly freeze-stored and cultured again. This contrasted strongly with sister cultures that were left untreated or transfected with pcDNA3-GFP, which reached senescence after 6-8 passages and barely resisted freezing (data not shown). Of note, fibroblasts generated from FIV-infected animals - regardless of whether immortalized or not - showed no evidence of FIV replication, as determined by weekly testing the supernatants for p25 capsid antigen, suggesting that they had been spared from infection or had stopped supporting detectable FIV replication as a result of culture (data not shown).

Transduction of pACTSV2 immortalized fibroblasts was carried out with viral particles produced in 293T cells and encapsidating the monocystronic vector carrying GFP alone (vGFP) or the bicistronic version delivering Env and GFP (vEnv-GFP) (Figure 1). For a preliminary assessment of the efficiency of transduction, the immortalized fibroblasts of an uninfected cat were exposed to serial 2-fold dilutions of a vGFP preparation and, 2 days later, analyzed for GFP expression by flow cytometry. The proportion of cells transduced proved clearly dose-dependent ranging from $10 \%$ with 12.5 transduction units (TU)/cell to over $90 \%$ with $200 \mathrm{TU} /$ cell. Importantly, transduced cells fluoresced at high levels (Figure 2A) even after several weeks in culture and when repeatedly stored frozen and recultured (data not shown), indicative of a stable transduction. Different batches of the vectors and fresh or thawed cells yielded similar results. Interestingly, for reasons that were not specifically addressed, immortalized fibroblasts proved consistently more prone to transduction (by a factor of approximately 5) than the nonimmortalized cells from which they derived (data not shown). 


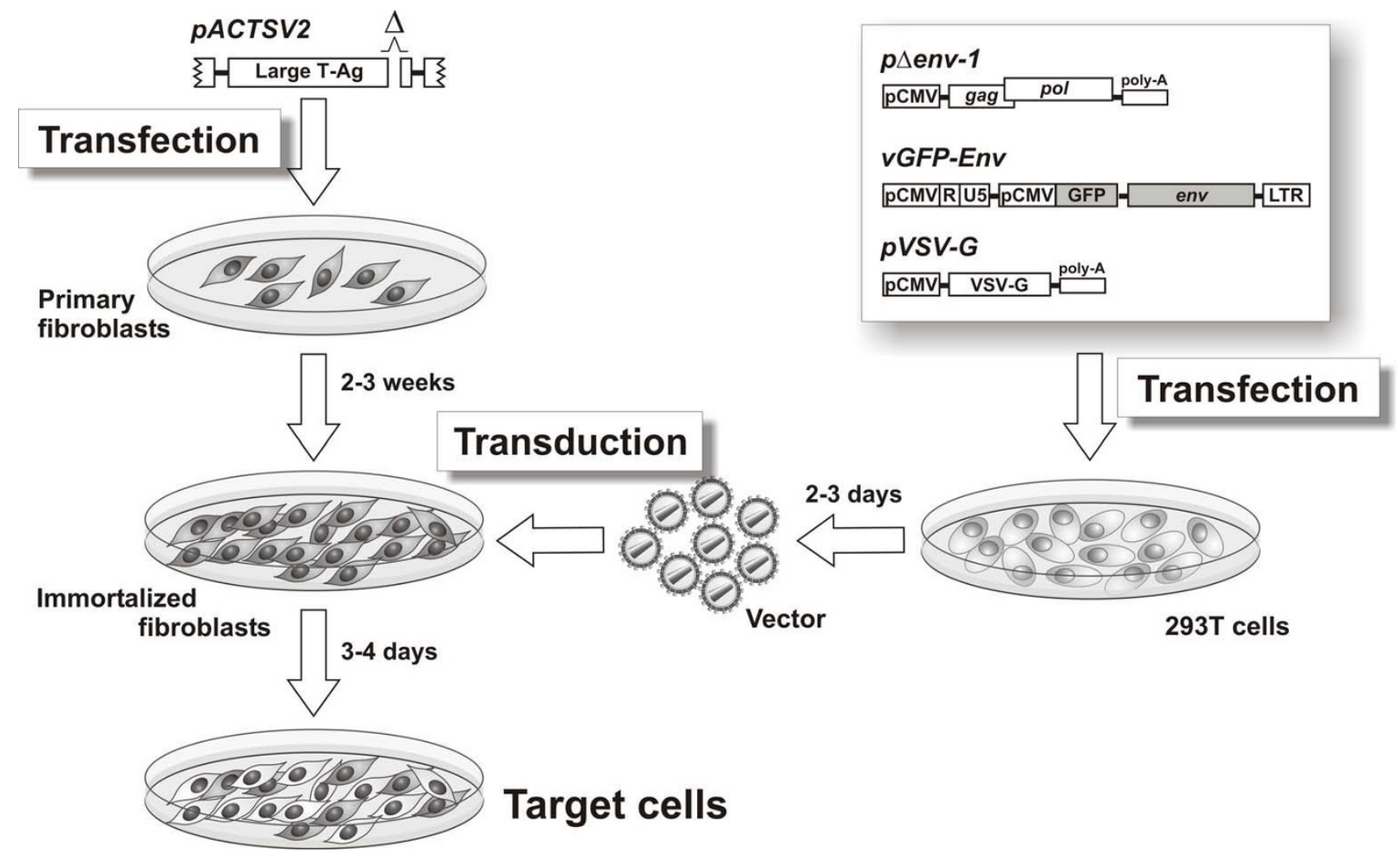

\section{Figure I}

Outline of target cell production. Primary feline fibroblasts were immortalized by transfection with plasmid pACTSV2, an SV40 genome encoding a functional small $\mathrm{T}$ antigen and an internally deleted large $\mathrm{T}$ antigen. The cells thus immortalized were then transduced with vesicular stomatitis virus glycoprotein G (VSV-G) pseudotyped particles produced in 293T cells and delivering target env gene and reporter GFP gene.

Unless differently stated, in the subsequent studies the immortalized fibroblasts expressing Env and GFP (Env ${ }^{+} \mathrm{GFP}^{+}$fibroblasts) used as targets were obtained by transducing with 100 TU vEnv-GFP per cell. Figure 2B shows the results obtained with this TU dose in one experiment in which we compared immortalized fibroblasts obtained from 4 cats, one uninfected and 3 chronically FIV infected, for susceptibility to transduction. Although at day 2 post-transduction the percentage of control cells expressing GFP alone (GFP+ fibroblasts) varied somewhat, transduction was successful with the cells of all cats. In addition, both the proportion of transduced cells and the extent they expressed Env had a clear tendency to increase with time, at least for the 3 weeks investigated (Figure 2C). Importantly, the Env expressed by Env ${ }^{+} \mathrm{GFP}^{+}$fibroblasts showed a precursor to surface glycoprotein ratio similar to that of cells chronically infected with FIV, indicating that it was processed correctly (Figure 2C).

The Env ${ }^{+}$GPP $^{+}$fibroblasts as a stimulus for expanding Envspecific CTL activity ex vivo

In this experiment we investigated if and how effectively the $\mathrm{Env}^{+} \mathrm{GFP}^{+}$fibroblasts could be used as a stimulus to expand Env-specific CTL activity ex vivo by comparing them with immortalized fibroblasts loaded with a pool of peptides covering the whole FIV Env. This was done by using the peripheral blood mononuclear cells (PBMCs) of BR, BS and CF, 3 chronically FIV infected cats known to possess substantial Env-specific CTL activity as a result of boosting with a DNA immunogen (see below). These PBMCs were co-cultured for 5 days with autologous immortalized fibroblasts that had been transduced with vEnv-GFP or had been pulsed for 1 hour with the pooled peptides or, as controls, with autologous $\mathrm{GFP}^{+}$fibroblasts or fibroblasts that had not been further manipulated. The PMBCs thus incubated were then tested for CTL activity against $\mathrm{Env}^{+} \mathrm{GFP}^{+}$fibroblasts. Lysis by PBMCs restimulated with autologous GFP+ fibroblasts or immortalized fibroblasts was at background levels (data not shown). The $\mathrm{Env}^{+} \mathrm{GFP}^{+}$fibroblasts caused an expansion of CTL activity readily measurable (Figure $3 \mathrm{~A}$ ) and substantially higher than the peptide-loaded counterparts (Figure 3B). As expected, either stimulus had no effect on the PBMCs of an uninfected cat used as control.

\section{The Env ${ }^{+} \mathrm{GFP}^{+}$fibroblasts as targets for measuring Env- specific CTL activity in acutely FIV infected cats}

Collectively, the above data had demonstrated that the Env $^{+} \mathrm{GFP}^{+}$fibroblasts expressed the target and reporter genes in stable and correct fashion. To test whether they 

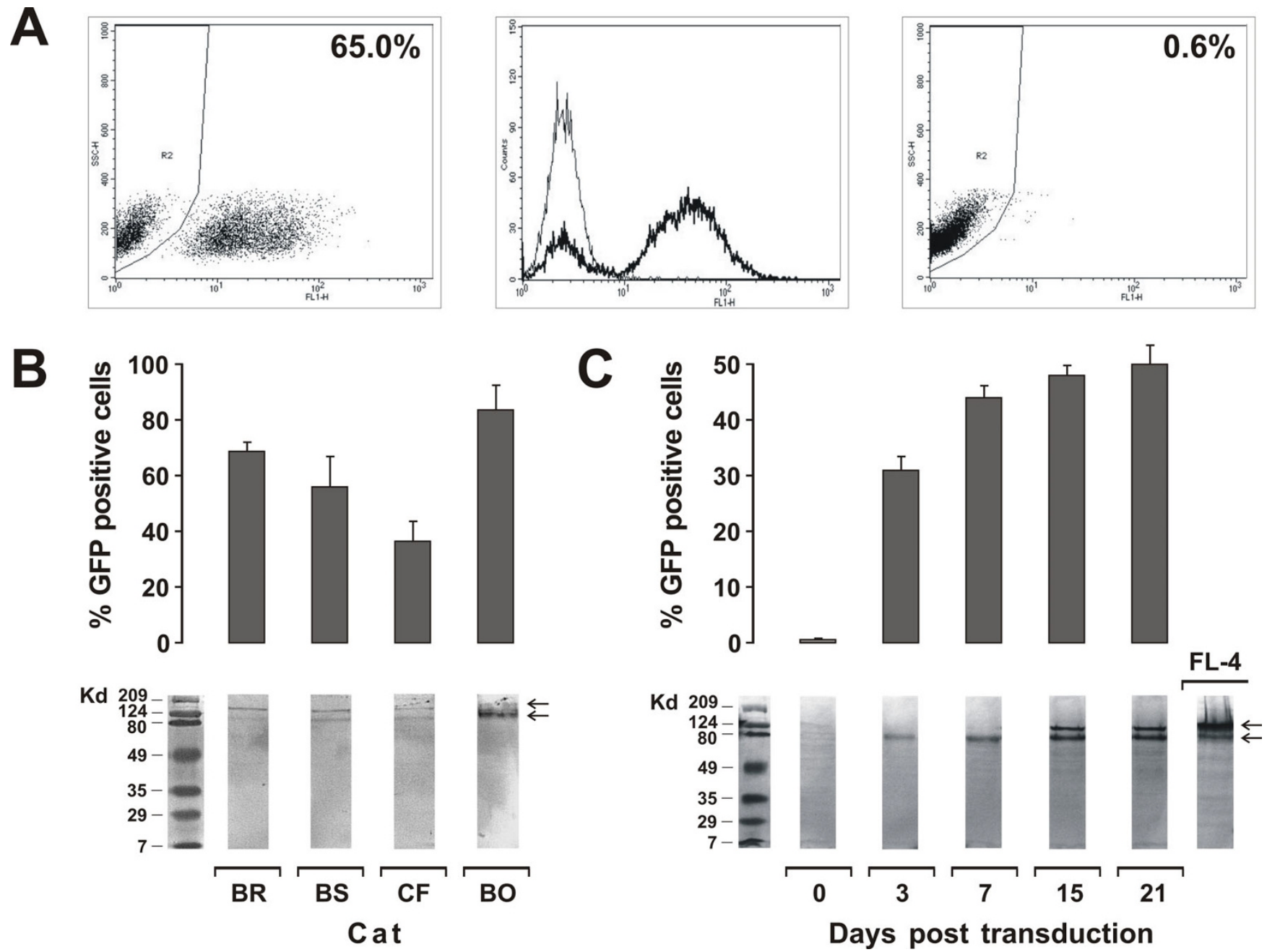

Figure 2

Efficiency and duration of transduction of immortalized feline fibroblasts by vGFP and vEnv-GFP. A: GFP expression by the fibroblasts of uninfected cat BO 2 days post-transduction with vGFP (50 TU/cell), as determined by flow cytometry. Left panel, percent GFP positive cells; middle panel, intensity of GFP expression; right panel, mock-transduced cells. B: Susceptibility of fibroblasts from 3 FIV chronically infected cats and I uninfected cat to transduction by vEnv-GFP at I00 TU/cell. Percent GFP positive cells (upper panel) and extent of Env expression (lower panel) were evaluated 2 days post-transduction by flow cytometry and western blot, respectively. C: GFP expression (upper panel) and Env expression (lower panel) by fibroblasts of one infected cat (BS) transduced with vEnv-GFP at 50 TU/cell, evaluated at the indicated times by flow cytometry and western blot, respectively. The arrows in panels $B$ and $C$ indicate the Env precursor (I35 Kd) and surface (95 Kd) glycoproteins. Bars represent SD calculated from 3 independent experiments. FL-4: Env expressed by the chronically FIV infected cell line FL-4.

were also suitable targets for CTL assays, we employed them in the F-CTL assay using, as effector cells, PBMCs collected from cats during acute FIV infection. Fibroblasts were harvested from SPF cats GN, GO, GP and GQ, processed as above, and frozen. The animals were then inoculated with a dose of FIV-Pet which produced the expected levels of viremia as determined starting 2 weeks postinfection. CTL activity was examined 2, 4, 8 and 12 weeks after infection. The test was carried out both with fresh PBMCs and with PBMCs that, prior to the F-CTL assay, were restimulated ex vivo with autologous $\mathrm{Env}^{+} \mathrm{GFP}^{+}$ fibroblasts as described above. Target cells were autologous $\mathrm{Env}^{+} \mathrm{GFP}^{+}$fibroblasts and, as controls, autologous $\mathrm{GFP}^{+}$fibroblasts and heterologous $\mathrm{Env}^{+} \mathrm{GFP}^{+}$fibroblasts; however, significant cell lysis was observed only with the former cells. As depicted by Figure 4, which shows the percent specific lysis values observed with the unstimulated PBMCs at effector:target (E:T) ratios of 1:10 and 1:50, in one cat CTL activity could already be detected 2 weeks post-infection. In the other animals, the test was first 

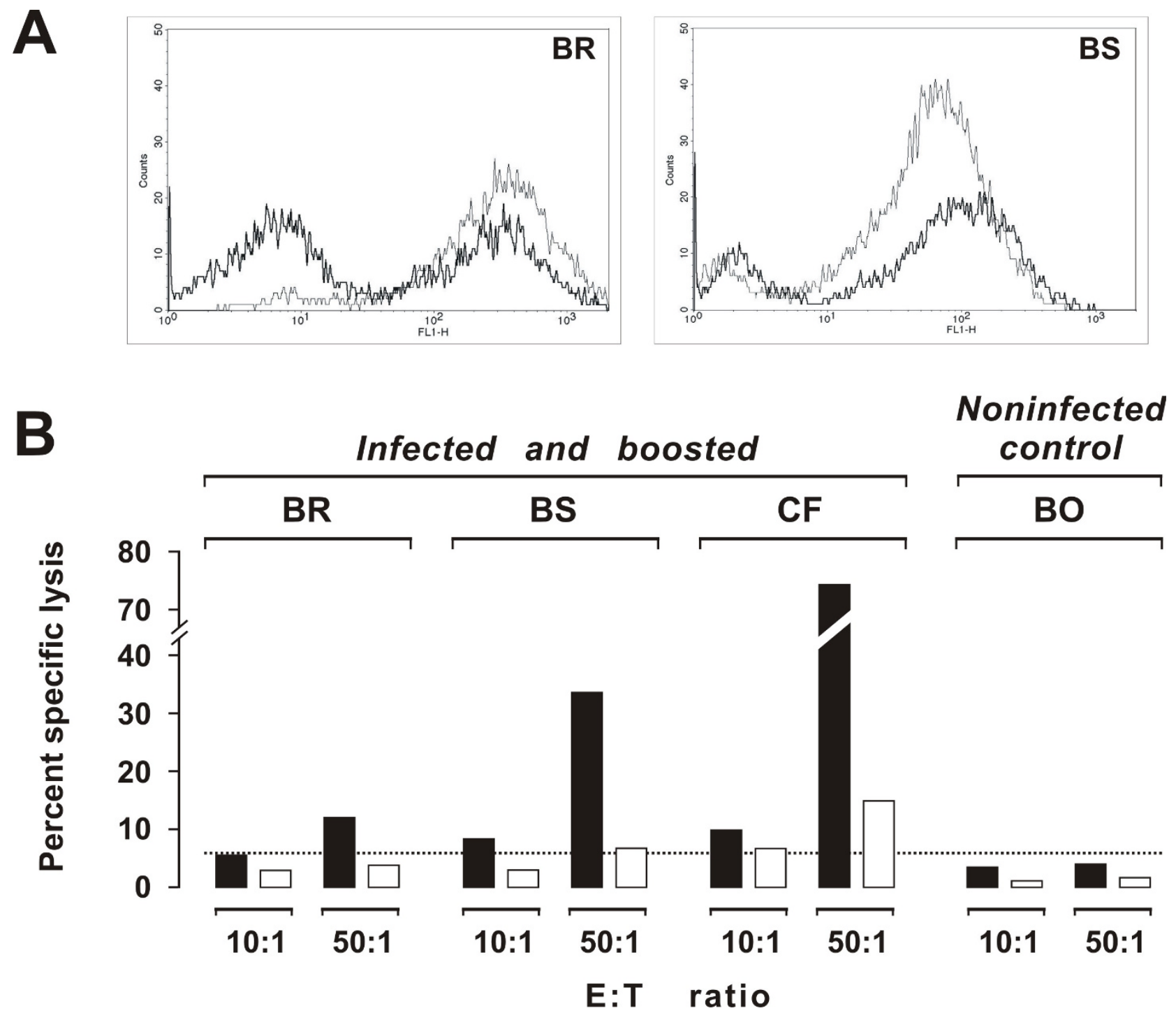

Figure 3

Flow cytometry plot of the CTL assay and comparative efficiency at restimulating CTL activity in vitro of Env transduced and Env peptides loaded immortalized fibroblasts. A: CTL activity following lymphocyte restimulation with Env-transduced immortalized fibroblasts as measured by F-CTL. Right and left panels show the results obtained with cats $B R$ and BS, respectively. The gray line indicates the target population incubated alone, the thick line indicates residual target cells after incubation with restimulated lymphocytes. B: Comparative efficiency of Env transduced and Env peptides-loaded immortalized fibroblasts at restimulating CTL activity. Prior to the assay, the effector cells (PBMCs of chronically FIV infected cats boosted with p-Env-GMCSF 18 weeks earlier) were restimulated with Env ${ }^{+} \mathrm{GFP}^{+}$fibroblasts (solid columns) or immortalized fibroblasts pulsed for I hour with pooled peptides covering the entire Env (empty columns).

found positive at 4 weeks post-infection, usually at the E:T ratio 50:1 only. At later times of infection, this picture did not change significantly, except for the fact that E:T ratio 10:1 yielded a measurable level of lysis more frequently than at earlier times. Percent lysis obtained with ex vivo restimulated PBMCs were only marginally higher than obtained with fresh PBMCs (data not shown), suggesting that in acutely infected cats Env-specific CTL activity cannot be significantly expanded ex vivo. Of note, cat GQ who exhibited the most prompt and robust CTL response developed levels of plasma viremia, proviral DNA in the $\mathrm{PBMC}$, and a reduction of circulating $\mathrm{CD} 4^{+} \mathrm{T}$-lymphocyte counts similar to the other animals (data not shown).

The Env ${ }^{+} G F P^{+}$fibroblasts as targets for measuring Envspecific CTL activity in chronically FIV infected cats In these experiments, $\mathrm{Env}^{+} \mathrm{GFP}^{+}$fibroblasts and F-CTL assay were used to measure anti-Env CTL in the PBMCs of three SPF cats infected with FIV-Pet 5 years earlier. As determined by periodical monitoring, these animals were 


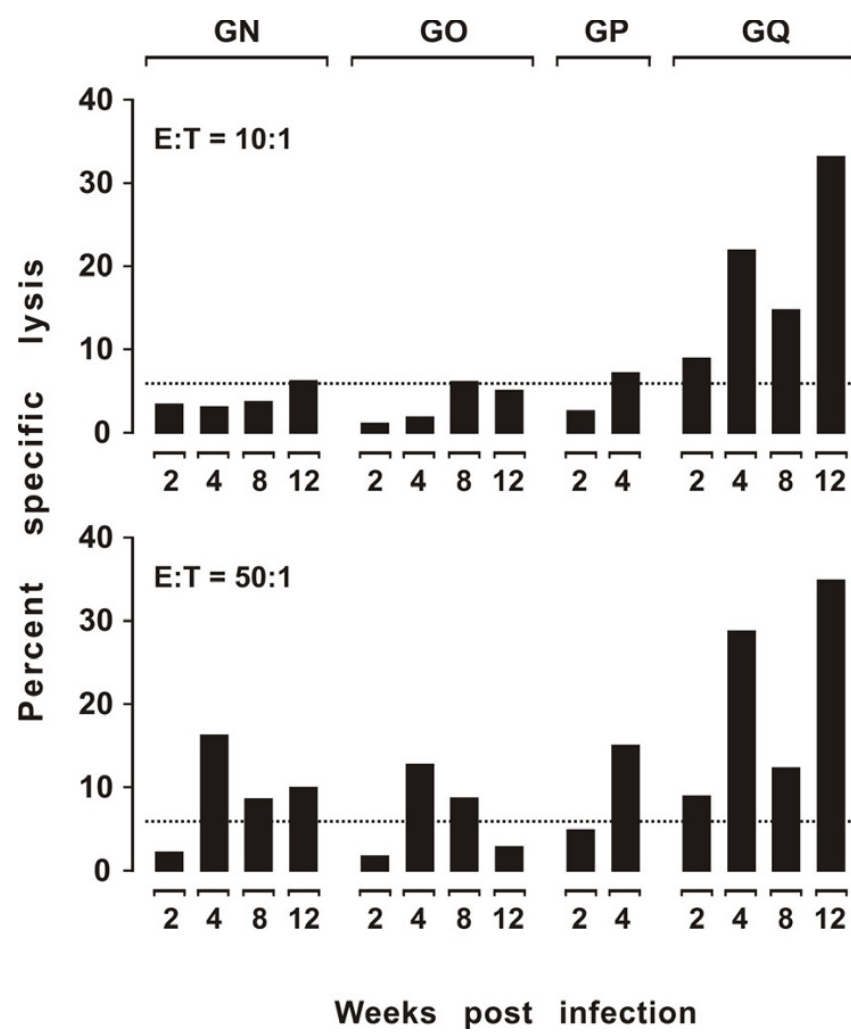

Figure 4

Env-specific CTL activity in acutely FIV infected cats. The PBMCs were obtained at the indicated times post-infection and used as effector cells without ex vivo restimulation. The dotted line indicates the cut-off value. Cat GP died 40 days post inoculation due to unexplained renal failure.

in a steady state phase of infection since they had stable or slightly fluctuating moderate levels of viremia, high FIV antibodies titers, and moderately reduced circulating CD4 ${ }^{+}$T-lymphocytes counts (data not shown). A fourth cat (BO), who had been inoculated exactly as the others but had remained consistently FIV negative, was used as noninfected control. Effector PBMCs were either used fresh or restimulated ex vivo by co-cultivation with autologous $\mathrm{Env}^{+} \mathrm{GFP}^{+}$fibroblasts; however, in no case they exhibited measurable CTL activity (time -8 weeks in Figure 5). An attempt was therefore done to boost anti-Env immune responses of the cats by DNA immunization. Starting 2 weeks after the above CTL assay, the 4 animals were given 3 doses of a plasmid encoding the Env of FIVPet and feline granulocyte-macrophage-colony stimulating factor (p-Env-GMCSF), 3 weeks apart, and after 2 further weeks again tested for CTL activity. As shown by Figure 5, the uninfected control cat showed no evidence that this immunization had elicited significant CTL activity. In contrast, all 3 FIV chronically infected animals exhibited robust CTL activity, ranging between $16 \%$ and $35 \%$ at E:T ratio 10:1. When the test was repeated 18 and
20 weeks after completion of DNA immunization, CTL activity was found to have substantially declined; however, it was generally in the measurable range (Figure 5) and, at least at week 18, it could be substantially expanded ex vivo (Figure 3 ). Incidentally, this immunization was also followed by a reduction of FIV RNA and proviral DNA loads and by an increment of circulating CD4 $\mathrm{T}^{+}$ lymphocytes, thus showing that it had exerted a beneficial effect on infection course (Pistello et al., manuscript in preparation).

\section{Discussion}

Measurement of CTL activity is considered of paramount importance for predicting protection from infection by lentiviruses and other pathogens $[1,3,4]$ but may be difficult due to a number of technical complications. In particular, production and use of suitable target cells generally needs expertise and facilities for using vaccinia vectors and radioactive compounds and, in non syngeneic hosts, it may be especially tricky due to the need for a constant source of MHC class 1 matched antigen-positive targets. In developing the CTL assay described here we took advantage of the method described by Kölsoy et al. [16] who, as target cells, used immortalized autologous feline primary fibroblasts infected with a recombinant vaccinia vector delivering the test antigen. Similarly infected primary feline fibroblasts have also been used as target cells but could be propagated in vitro for maximum 1-2 months before undergoing senescence and eventually dying off [17-19]. In their study, Kölsoy et al. demonstrated that it is possible to obtain an unlimited source of

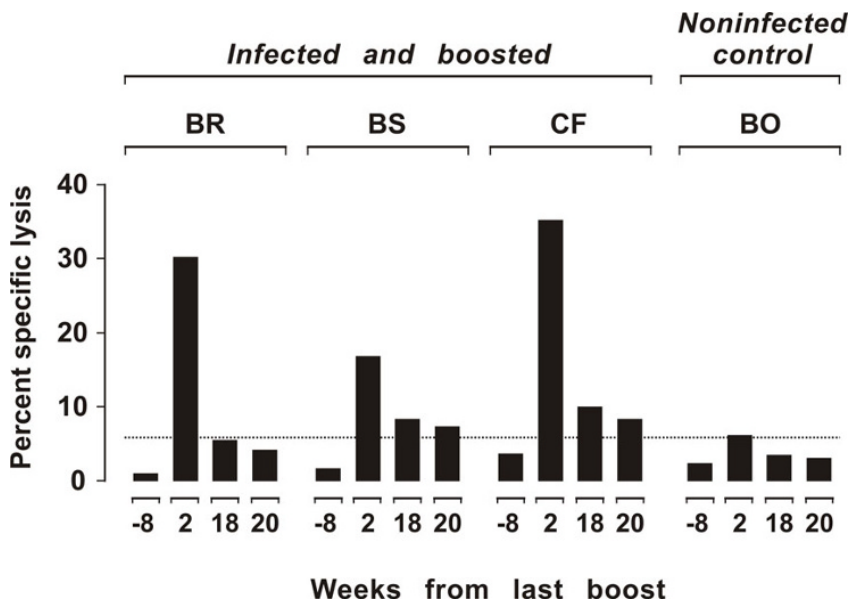

Figure 5

Env-specific CTL activity in chronically FIV infected cats before and after boosting with a DNA immunogen. The effector PBMCs were obtained at the indicated times and used at E:T ratio I0:I after ex vivo restimulation with $\mathrm{Env}^{+} \mathrm{GFP}^{+}$fibroblasts. The dotted line indicates the cutoff value. 
target cells retaining the same MHC class I phenotype as the cells of origin by immortalizing fibroblasts with replication competent SV40. To avoid full transformation and minimize the risk of abnormal self-antigen expression, we have instead used a molecular clone of SV40, rendered unable to replicate through an internal deletion in the large T-antigen, which immortalized primary feline fibroblasts as efficiently as wild-type SV40 (data not shown). This clone had previously been shown to transform established cell lines, such as NIH-3T3 cells, but to be unable to transform primary rat embryo fibroblasts [20-22].

Because we wished to test immortalized fibroblasts as a tool for measuring Env-specific CTL activity in FIV infected and vaccinated cats, we attempted to infect these cells with FIV, with no success. Fibroblasts from FIV infected cats also proved consistently virus-negative, thus excluding the possibility of using direct FIV infection as a means of obtaining the desired target cells. We therefore exploited a bicistronic vesicular stomatitis virus glycoprotein G (VSV-G) coated vector derived in our laboratory from FIV but carrying very little of the original virus, vector that had been previously shown to effectively introduce transgenes into primary cells of both feline and nonfeline origin [15]. The vector used carried the Env of FIV-Pet and GFP as target and reporter transgene, respectively. Immortalized fibroblasts transduced at high multiplicity with this vector were approximately 90\% positive for both transgenes and proved valuable both as target cells for use in a F-CTL assay and as a stimulus for expanding CTLs ex vivo.

Differently from the methods that rely on vaccinia virus infection or transfection or on peptide loading used in traditional ${ }^{51} \mathrm{Cr}$ assays $[5,6]$ and in the more recently described (F-CTL) assays [7-12], this method generates target cells stably expressing the antigen of interest and the reporter molecule. This has been achieved by using a bicistronic vector that $i$ ) does not require the containment procedures needed for the recombinant vaccinia viruses that are frequently used to introduce transgenes in target cells, ii) overcomes the intrinsic resistance to transfection of primary and non-adherent cells, $\mathrm{iii}$ ) delivers two large transgenes for up to $7 \mathrm{Kbs}$, iv) stably transduces cells, thus permitting to use the same batch of targets in follow-up studies, minimizing inter-assay variation, $v$ ) allows transduction of cells irrespective of animal species; vi) is replication incompetent, making it unnecessary ultraviolet irradiation or other treatments of the target cells to prevent infection transmission to effector cells, and last but not least, vii) permits the use of F-CTL assays. While most other CTL assays detect radioisotopes or cell dyes released from the target cell population in bulk and may have a high background noise due to spontaneous release of the markers, F-CTL assays rely on the actual enumeration of target cells made fluorescent by GFP, a protein that is not secreted by the cells and does not fluoresce if degraded or improperly folded in dying cells, and with the use of specific cell-specific markers may also permit phenotyping of the effector cells [6,9].

A limitation of the present study is that the F-CTL assay was not directly compared to established techniques. However, its validity was assessed by evaluating FIV Envspecific CTL activity in both acutely and chronically FIVinfected cats. The method proved valuable in either situation. During the early stages of infection, characterized by acute viral replication and vigorous cell-mediated immune responses [18,23], the assay detected levels of Env-specific CTL activity in the PBMCs of infected cats similar to those previously reported with ${ }^{51} \mathrm{Cr}$ CTL assays using SV40-immortalized or primary feline fibroblasts infected by recombinant vaccinia viruses $[18,24]$, with no need for the effector cells to be restimulated ex vivo, a procedure frequently necessary when using the standard $\mathrm{Cr}^{51}$ assay [25]. In contrast, in the chronically infected cells we detected no measurable Env-specific CTL activity even when the effector PBMCs were restimulated ex vivo. This may have reflected the fact that, especially in the periphery [18], anti-FIV Env cell-mediated immune responses tend to wane with time of infection [26-28], or immunosenescence of the cats who were 10 year-old when tested [29], or a combination of these factors [30]. In any case, administration to the chronically infected cats of a DNA vaccine encoding the Env of the infecting FIV and feline granuloyte macrophage-colony stimulating factor (GM-CSF), a cytokine known to induce dendritic cell proliferation and improve antigen presentation [31], with a protocol that had elicited fairly good cell-mediated immune responses when tested in a prior prophylactic study (Pistello et al., manuscript in preparation), brought back substantial levels of CTL activity. CTL activity showed a maximum 2 weeks after completion of the vaccination schedule and then gradually decreased as previously observed for other FIV antigens $[18,25]$, but in $2 / 3$ animals it remained detectable for at least 20 weeks without the need for restimulating ex vivo their PBMC. Interestingly, this correlated with a beneficial effect of the vaccine on infection course, as demonstrated by a decrease of viral RNA in plasma and proviral load in the PBMCs and an increase of circulating CD4+ T-lymphocytes (Pistello, manuscript in preparation). Of note, vaccination on an uninfected control cat elicited a modest CTL response that exceeded the cut-off only at an early post-vaccination time point only, suggesting that the vigorous CTL activity brought forth by vaccination in the chronically infected cats was mostly due to a recall of pre-existing immunity rather than to ex novo induction. 


\section{Conclusion}

The F-CTL assay described, using immortalized feline fibroblasts stably transduced with the test antigen and the GFP reporter as target cells, has proven valuable in evaluating Env-specific CTL activity in FIV infected and vaccinated cats. Given the versatility of the bicistronic vector used for producing target cells, the method has the potential to be used for a wide array of antigens and hosts.

\section{Methods}

\section{Plasmids and vectors}

The GFP encoding plasmid pcDNA3-GFP was previously described [25]. Plasmid pACTSV2 encoding a replication defective SV40 genome with a functional small $\mathrm{T}$ and a large $\mathrm{T}$ antigen mutated by an internal deletion of 43 amino acids [20] was a kind gift of Dr. Mauro Tognon (University of Ferrara, Italy). Construction of the bicistronic vEnv-GFP and monocystronic vGFP vectors, derived from molecular clone p34TF10 of FIV-Pet, and of the packaging ( $\mathrm{p} \Delta \mathrm{env} 1)$ and VSV G-protein (pVSV-G) plasmids has been described [15]. Briefly, vEnv-GFP delivers the target Env of FIV-Pet and the reporter GFP placed under the control of the FIV LTR and the cytomegalovirus promoter, respectively (Figure 1). vGFP has the same architecture except that it encodes the reporter alone. Pseudotyped vector particles were generated in human epithelial 293T cells that were co-transfected with $\mathrm{p} \Delta \mathrm{env}$, pVSV-G and either vEnv-GFP or vGFP, exactly as described [15]. Two days later, approximately $80 \%$ of the cells were GFP positive by FACS analysis, regardless of whether exposed to vEnv-GFP or vGFP. The pseudotyped virus preparations used for transduction consisted of 293T cell culture supernatants harvested on day 2, filtered through a $0.45 \mu \mathrm{m}$ filter (Nalge Europe, Neerijse, Belgium), and titered for TU in 293T cells as described [15]. They were either used immediately or stored in aliquots at $-80^{\circ} \mathrm{C}$ until use, with no appreciable differences in transduction efficiency.

\section{Animals}

The SPF cats (IFFA Credo, Lyon, France) used were housed individually in our climatized animal facility in accordance with European Community guidelines, had ad libitum access to fresh water and a proprietary brand of cat food, and were sedated with ketamine/diazepam intramuscularly prior to any procedure. Cats GN, GO, GP and GQ were FIV infected after entering the study (acutely infected cats). Infection was performed intraperitoneally when 18-month old with $1 \mathrm{ml}$ of a stock of pooled plasma from FIV-Pet infected cats diluted to contain 10 cat infectious doses $50 \%$ per $\mathrm{ml}$. Three further cats (BR, BS and CF) were already infected when the study started (chronically infected cats). These had been infected exactly as above 5 years earlier and were 10-year old at the start of the study. An additional 10-year old cat (BO) that had been simi- larly inoculated but had escaped infection by all parameters served as uninfected control for the latter animals. Because the chronically infected cats showed no detectable Env-specific CTL activity (see below), they were boosted by DNA immunization with p-Env-GMCSF, a plasmid expressing the Env of FIV-Pet and feline GM-CSF, as an immunoadjuvant (Pistello et al., manuscript in preparation). This was inoculated intramuscularly at doses of $300 \mu \mathrm{g}(300 \mu \mathrm{l})$ into both hind legs $(150 \mu \mathrm{l} /$ site $)$ 20 minutes after rubbing the shaved skin with Aldara (Graceway Pharmaceuticals, Bristol, TN, USA), a topical Imiquimod containing cream known to exert an adjuvant effect. Infection of cats was monitored as described [25] by testing for presence and loads of FIV RNA in plasma and proviral DNA in the PBMCs, anti-FIV antibody in serum and counting peripheral $\mathrm{CD}^{+}$and $\mathrm{CD}^{+}{ }^{+} \mathrm{T}-\mathrm{lym}-$ phocyte.

\section{Preparation of target cells}

Primary fibroblasts were obtained from skin biopsies of the study cats with sterile, $4 \mathrm{~mm}$ diameter, disposable skin biopsy punches (Steifel Laboratories, Segrate, Italy) and cultured in 6-well plates with minimum essential medium- $\alpha$ (Sigma-Aldrich, Milan, Italy) supplemented with ribonucleosides and deoxyribonucleosides, 10\% fetal bovine serum (FBS; Sigma-Aldrich), 200 U/ml penicillin and streptomycin (Eurobio, Labtek, Milan, Italy) and $2 \mathrm{mM}$ L-glutamine (Sigma-Aldrich). Around day 20, when the cultures were confluent, the cells were immortalized by transfecting $4-5 \times 10^{4}$ cells, seeded the day before in $10 \mathrm{~cm}$ Petri dishes, with $2 \mu \mathrm{g}$ pACTSV2 $[20,21]$ and using a calcium phosphate method previously described [15]. This led to the appearance of typical signs of cell immortalization (i.e. unlimited replication, loss of inhibition contact and growth in low FBS medium) in approximately one week and to generalized immortalization in 10 to 15 days. The fibroblasts thus immortalized were then seeded into 6 -well plates at $3 \times 10^{5}$ cells/well and, 18 hours later, transduced with $3 \times 10^{6} \mathrm{TU}$ of vEnvGFP (Env ${ }^{+} \mathrm{GFP}^{+}$fibroblasts) or, as antigen control, with a similar dose of vGFP (GFP+ fibroblasts). Expression of the transgenes was monitored 2 days post-transduction and then twice weekly for up to 4 weeks by flow cytometry (GFP) and Western blot (Env). Finally, the target cells were expanded and frozen for further use.

\section{Analysis of the target cells for Env expression}

Env expression by the target cells was evaluated by Western blot analysis. Briefly, $1 \times 10^{5}$ cells were lysed in $100 \mu \mathrm{l}$ lysis buffer $(10 \mathrm{mM}$ Tris- $\mathrm{HCl} \mathrm{pH} 7.5,150 \mathrm{mM} \mathrm{NaCl}, 2$ mM EDTA, $0.5 \%$ Nonidet P-40), mixed with $2 \times$ loading buffer, and loaded $(20 \mu \mathrm{l})$ on a $10 \%$ polyacrylamide gel (Bio-Rad Laboratories, Milan, Italy). Proteins were transferred onto a nytran C-extra nitrocellulose membrane (General Electric Healthcare, Milan, Italy) and reacted 
with the anti-FIV surface glycoprotein monoclonal antibody vpg71.2, kind gift of Dr. Brian Willett, University of Glasgow, Glasgow, UK. Detection was done with a rabbit anti-mouse IgG horseradish peroxidase-conjugated monoclonal antibody (Sigma-Aldrich). The FIV chronically infected feline lymphoid cell line FL-4 [32] was used as positive control.

\section{Effector cells}

Effector cells were PBMCs separated from $20 \mathrm{ml}$ of EDTA or citrate anticoagulated blood of the study cats on Ficoll/ Hystopaque 1077 (Sigma-Aldrich), washed in PBS, viability counted with trypan blue, and suspended in RPMI 1640 (Sigma Aldrich) supplemented with $2 \mathrm{mM} \mathrm{L-}$ glutamine, $200 \mathrm{U} / \mathrm{ml}$ penicillin and streptomycin and $10 \%$ FBS. PBMCs were used as such or after ex vivo restimulation. For restimulation, $5 \times 10^{6}$ PBMCs were cultured in RPMI 1640 supplemented as above in 6-well plates that had been seeded with $1 \times 10^{5} \mathrm{Env}^{+} \mathrm{GFP}^{+}$or, as a negative control, GFP+ fibroblasts 1 day earlier. After partial substitution of the culture medium with fresh medium containing $20 \mathrm{U} / \mathrm{ml}$ human interleukin-2 (Roche Diagnostics, Milan Italy) at day 3, the restimulated cells were harvested on day 5 , viability counted, and suspended as above. In one experiment, restimulation was also carried out by incubating the PBMCs with immortalized fibroblasts that had been loaded for 1 hour with $10 \mu \mathrm{M}$ (final concentration) pooled 21-mer peptides overlapping by 11 amino acids and encompassing the entire sequence of the FIV-Pet Env. The peptides had been synthesized by Espikem (Florence, Italy) with fmoc chemistry and were $>95 \%$ pure.

\section{F-CTL assay}

The day before the assay, $1 \times 10^{4} \mathrm{Env}^{+} \mathrm{GFP}^{+}$fibroblasts and, as negative control, GFP+ fibroblasts derived from at least two distinct cats were individually seeded onto a 48well plate and incubated overnight to allow complete adherence. PBMCs, either freshly separated or restimulated in vitro, were added at E:T ratios of 10:1 and 50:1 onto autologous and heterologous fibroblasts. After overnight incubation at $37^{\circ} \mathrm{C}$ in a humidified $\mathrm{CO}_{2}$ atmosphere, the PBMCs were removed, and the adherent cells extensively washed, trypsinized and fixed for FACS analysis. Analysis was performed using a FACScan cytometer (Becton Dickinson). The forward scatter (FSC) threshold was set to measure nonviable cells as well and to acquire $1 \times 10^{6}$ events/sample. Cellular debris were then left out by gating in FSC-SSC dot plots during data acquisition. Numbers of viable fluorescent cells were plotted in a number of events/fluorescence intensity (FL-1) histogram, and acquired data were analyzed using CellQuest Pro software (version 3.4). Samples were analyzed in triplicate and percent specific anti-Env CTL activity was calculated using the formula: (\% fluorescent target cells incubated alone - \% fluorescent target cells incubated with autologous effector cells) - (\% fluorescent target cells incubated alone - \% fluorescent target cells incubated with heterologous effector cells) $/ \%$ fluorescent target cells incubated alone $\times 100$. In preliminary studies aimed at evaluating nonspecific target cell lysis, PBMCs were also incubated with autologous and heterologous $\mathrm{GFP}^{+}$positive cells. Nonspecific lysis (background), calculated using cat BR, BS, CF, and BO cells in three independent experiments, averaged $2.9 \%$, and standard deviation (SD) was 1.0. Thus, values of lysis above $6 \%$ (i.e. background $+3 \times$ $\mathrm{SD})$ were considered positive.

\section{Authors' contributions}

$\mathrm{FB}$ and $\mathrm{EZ}$ devised the experiments, produced the vector constructs and performed in vitro and in vivo tests. FC established the primary cell lines and together with EZ immortalized and transduced the cells. AM vaccinated and monitored clinical conditions and hematological parameters of animals. GF helped to the set up the F-CTL assay and carried out flow cytometry analyses together with EZ and FC. MB and MP wrote the manuscript. All authors read and approved the final manuscript.

\section{Acknowledgements}

This study was supported by Ministero dell'Istruzione, dell'Università e della Ricerca, grants PRIN 2005 and 2007, and Ministero della Salute - Istituto Superiore di Sanità, "VI ${ }^{\circ}$ Programma Nazionale di Ricerca sull'AIDS 2006" and "Progetto Nazionale AIDS - ICAV".

This work was funded by the grants indicated above and held by MP.

\section{References}

I. Doherty PC, Turner S): The challenge of viral immunity. Immunity 2007, 27:363-365.

2. Koup RA, Safrit JT, Cao Y, Andrews CA, McLeod G, Borkowsky W, Farthing C, Ho DD: Temporal association of cellular immune responses with the initial control of viremia in primary human immunodeficiency virus type I syndrome. J Virol I994, 68:4650-4655.

3. Letvin NL: Correlates of immune protection and the development of a human immunodeficiency virus vaccine. Immunity 2007, 27:366-369.

4. Deeks SG, Walker BD: Human immunodeficiency virus controllers: mechanisms of durable virus control in the absence of antiretroviral therapy. Immunity 2007, 27:406-416.

5. Brunner KT, Mauel J, Cerottini JC, Chapuis B: Quantitative assay of the lytic action of immune lymphoid cells on $5 \mathrm{I}-\mathrm{Cr}$ labelled allogeneic target cells in vitro; inhibition by isoantibody and by drugs. Immunology 1968, 14:181-196.

6. Shacklett BL: Beyond ${ }^{5} \mathrm{Cr}$ release: new methods for assessing HIV-I-specific CD8+ T cell responses in peripheral blood and mucosal tissues. Clin Exp Immunol 2002, I 30: 172-182.

7. Chahroudi A, Silvestri G, Feinberg MB: Measuring T cell-mediated cytotoxicity using fluorogenic caspase substrates. Methods 2003, 31: 120-126.

8. Chen K, Chen L, Zhao P, Marrero L, Keoshkerian E, Ramsay A, Cui Y: FL-CTL assay: fluorolysometric determination of cellmediated cytotoxicity using green fluorescent protein and red fluorescent protein expressing target cells. J Immunol Methods 2005, 300:100-II4.

9. Godoy-Ramirez K, Makitalo B, Thorstensson R, Sandstrom E, Biberfeld G, Gaines H: A novel assay for assessment of HIV-specific cytotoxicity by multiparameter flow cytometry. Cytometry $A$ 2005, 68:71-80. 
10. Kienzle N, Olver S, Buttigieg K, Kelso A: The fluorolysis assay, a highly sensitive method for measuring the cytolytic activity of $\mathbf{T}$ cells at very low numbers. J Immunol Methods 2002, 267:99-108.

II. van Baalen CA, Kwa D, Verschuren EJ, Reedijk ML, Boon AC, de Mutsert G, Rimmelzwaan GF, Osterhaus AD, Gruters RA: Fluorescent antigen-transfected target cell cytotoxic $T$ lymphocyte assay for ex vivo detection of antigen-specific cell-mediated cytotoxicity. J Infect Dis 2005, 192: I I83-1190.

12. Wang Y, Korkeamaki M, Vainio O: A novel method to analyze viral antigen-specific cytolytic activity in the chicken utilizing flow cytometry. Vet Immunol Immunopathol 2003, 95: I-9.

13. Dunham S, Jarrett O: FIV as a model for AIDS vaccine studies. In Animal Models of HIV Infection and Control Edited by: Friedman H, Bendinelli M, Specter S. New York: Springer; 2006:293-332.

14. Sparger EE: FIV as a model for HIV: an overview. In Animal Models of HIV Infection and Control Edited by: Friedman $\mathrm{H}$, Bendinelli $\mathrm{M}$, Specter S. New York: Springer; 2006:149-237.

15. Pistello M, Vannucci L, Ravani A, Bonci F, Chiuppesi F, Del Santo B, Freer G, Bendinelli M: Streamlined design of a self-inactivating feline immunodeficiency virus vector for transducing ex vivo dendritic cells and T lymphocytes. Genet Vaccines Ther 2007, 5:8.

16. Köksoy S, Phipps AJ, Hayes KA, Mathes LE: SV40 Immortalization of feline fibroblasts as targets for MHC-restricted cytotoxic T-cell assays. Vet Immunol Immunopathol 200I, 79:285-295.

17. Beatty JA, Willett BJ, Gault EA, Jarrett O: A longitudinal study of feline immunodeficiency virus-specific cytotoxic T lymphocytes in experimentally infected cats, using antigen-specific induction. / Virol 1996, 70:6199-6206.

18. Flynn JN, Dunham S, Mueller A, Cannon C, Jarrett O: Involvement of cytolytic and non-cytolytic $T$ cells in the control of feline immunodeficiency virus infection. Vet Immunol Immunopathol 2002, 85: I59-170.

19. Kooistra LH, Splitter GA: Killer cells of feline leukemia virusand feline sarcoma virus-infected transformed cells: the role of NK, ADCC, and in vitro generated cytotoxic cells. Cell Immunol 1985, 94:466-479.

20. Fischer-Fantuzzi L, Scheidtmann KH, Vesco C: Biochemical properties of a transforming nonkariophilic $T$ antigen and SV40. Virology 1986, I 53:87-95.

21. Fischer-Fantuzzi L, Vesco C: Deletion of $\mathbf{4 3}$ amino acids in the NH2-terminal half of the large tumor antigen of simian virus 40 results in a non-karyophilic protein capable of transforming established cells. Proc Natl Acad Sci USA 1985, 82: I89|-I895.

22. Fischer-Fantuzzi L, Vesco C: A nonkaryophilic T antigen of SV40 can either immortalize or transform rodent cells, and cooperates better with cytoplasmic than with nuclear oncoproteins. Oncogene Res 1987, I(3):229-242.

23. Hosie MJ, Beatty JA: Vaccine protection against feline immunodeficiency virus: setting the challenge. Aust Vet $J$ 2007, 85:302-303.

24. Hayes KA, Köksoy S, Phipps AJ, Buck WR, Kociba GJ, Mathes LE: Lentivirus-specific cytotoxic $T$-lymphocyte responses are rapidly lost in thymectomized cats infected with feline immunodeficiency virus. J Virol 2005, 79:8237-8242.

25. Pistello M, Bonci F, Flynn JN, Mazzetti P, Isola P, Zabogli E, Camerini V, Matteucci D, Freer G, Pelosi P, Bendinelli M: AIDS vaccination studies with an ex vivo feline immunodeficiency virus model: analysis of the accessory ORF-A protein and DNA as protective immunogens. J Virol 2006, 80:8856-8868.

26. Flynn JN, Beatty JA, Cannon CA, Stephens EB, Hosie MJ, Neil JC, Jarrett $O$ : Involvement of gag- and env-specific cytotoxic $T$ lymphocytes in protective immunity to feline immunodeficiency virus. AIDS Res Hum Retroviruses 1995, II: I I07-III 3.

27. Matteucci D, Pistello M, Mazzetti P, Giannecchini S, Isola P, Merico A, Zaccaro L, Rizzuti A, Bendinelli M: AIDS vaccination studies using feline immunodeficiency virus as a model: immunisation with inactivated whole virus suppresses viraemia levels following intravaginal challenge with infected cells but not following intravenous challenge with cell-free virus. Vaccine 18:119-130.

28. Pistello M, Matteucci D, Bonci F, Isola P, Mazzetti P, Zaccaro L, Merico A, Del Mauro D, Flynn N, Bendinelli M: AIDS vaccination studies using an ex vivo feline immunodeficiency virus model: protection from an intraclade challenge administered sys- temically or mucosally by an attenuated vaccine. J Virol 2003 , 77:10740-10750

29. Campbell DJ, Rawlings JM, Koelsch S, Wallace J, Strain JJ, Hannigan BM: Age-related differences in parameters of feline immune status. Vet Immunol Immunopathol 2004, 100:73-80.

30. George JW, Pedersen NC, Higgins J: The effect of age on the course of experimental feline immunodeficiency virus infection in cats. AIDS Res Hum Retroviruses 1993, 9:897-905.

31. Arellano M, Waller KE: Granulocyte-macrophage-colony-stimulating factor and other cytokines: as adjuncts to cancer immunotherapy, stem cell transplantation, and vaccines. Curr Hematol Rep 2004, 3:424-431.

32. Yamamoto JK, Ackley CD, Zochlinski H, Louie H, Pembroke E, Torten M, Hansen H, Munn R, Okuda T: Development of IL-2independent feline lymphoid cell lines chronically infected with feline immunodeficiency virus: importance for diagnostic reagents and vaccines. Intervirology 199|, 32:36I-375.

Publish with Bio Med Central and every scientist can read your work free of charge

"BioMed Central will be the most significant development for disseminating the results of biomedical research in our lifetime. "

Sir Paul Nurse, Cancer Research UK

Your research papers will be:

- available free of charge to the entire biomedical community

- peer reviewed and published immediately upon acceptance

- cited in PubMed and archived on PubMed Central

- yours - you keep the copyright 